\title{
BMJ Open Cost-effectiveness of stroke care in Aboriginal and non-Aboriginal patients: an observational cohort study in the Northern Territory of Australia
}

\author{
Yuejen Zhao, ${ }^{1}$ Steven Guthridge, ${ }^{1,2}$ Henrik Falhammar, ${ }^{2,3,4,5}$ Howard Flavell, ${ }^{2,3}$ \\ Dominique A Cadilhac ${ }^{6,7,8}$
}

To cite: Zhao Y, Guthridge S, Falhammar $\mathrm{H}$, et al. Costeffectiveness of stroke care in Aboriginal and non-Aboriginal patients: an observational cohort study in the Northern Territory of Australia. BMJ Open 2017;7:e015033. doi:10.1136/ bmjopen-2016-015033

- Prepublication history for this paper is available online. To view these files please visit the journal online (http://dx.doi. org/10.1136/bmjopen-2016015033)

Received 7 November 2016 Revised 7 April 2017 Accepted 21 April 2017

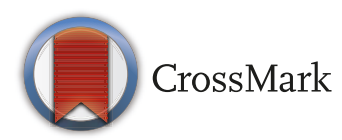

For numbered affiliations see end of article.

Correspondence to

Dr Yuejen Zhao;

yuejen.zhao@nt.gov.au

\section{ABSTRACT}

Objective To assess cost-effectiveness of stroke care for Aboriginal compared with non-Aboriginal patients in the Northern Territory (NT), Australia.

Design Cost-effectiveness analysis using data from a cohort-based follow-up study of stroke incidents. Setting Public hospitals in the NT from 1992 to 2013. Participants Individual patient data were extracted and linked from the hospital inpatient and primary care information systems.

Outcome measures Incremental cost-effectiveness ratios were calculated and assessed graphically. Survival time was used to measure effectiveness of stroke care, in comparison with the net costs per lifeyear gained, from a healthcare perspective, by applying multivariable models to account for time-dependent confounding.

Results 2158 patients with incident stroke were included (1171 males, 1178 aged $<65$ years and 966 from remote areas). 992 patients were of Aboriginal origin $(46.0 \%$, disproportionately higher than the population proportion of $27 \%$ ). Of all cases, $42.6 \%$ were ischaemic and $29.8 \%$ haemorrhagic stroke. Average age of stroke onset was 51 years in Aboriginal, compared with 65 years in non-Aboriginal patients $(p<0.001)$. Aboriginal patients had $71.4 \%$ more hospital beddays, and $7.4 \%$ fewer procedures than non-Aboriginal patients. Observed health costs averaged $\$ A 50400$ per Aboriginal compared with $\$ A 33700$ per non-Aboriginal patient $(p<0.001)$. The differential costs and effects for each population were distributed evenly across the incremental cost-effectiveness plane threshold line, indicating no difference in cost-effectiveness between populations. After further adjustment for confounding and censoring, cost-effectiveness appeared greater for Aboriginal than non-Aboriginal patients, but this was not statistically significant $(p=0.25)$.

Conclusions Stroke care for the NT Aboriginal population is at least as cost-effective as the nonAboriginal population. Stroke care presents worthwhile and equitable survival benefits for Aboriginal patients in remote communities, notwithstanding their higher level burden of disease. These findings are relevant for healthcare planning and policy development regarding equal access to stroke care for Aboriginal patients.
Strengths and limitations of this study

- This study included a large sample size of Aboriginal patients with stroke relative to the non-Aboriginal patients over a 21 -year period, using linked patient records across multiple sources of data.

- The methodology informs cost-effectiveness analysis for both patients and providers in real-world settings, which used non-randomised observational data and focused on more relevant health policy issues.

- The results are most relevant to Aboriginal populations living in remote locations, who experience socioeconomic disadvantage and high burden of disease in a high-income country.

- The lifetime stroke costs were based on health service use, which did not cover costs associated with the loss of quality of life among stroke survivors.

\section{INTRODUCTION}

Worldwide, stroke has a substantial impact on the health of populations and on health systems, and about 16.9 million people suffered first-ever stroke, with 5.9 million stroke-related deaths in 2010. ${ }^{1}$ Approximately 51000 Australians experience a new or recurrent stroke each year, and stroke is a national priority since it is a leading cause of death and disability. ${ }^{2}$ The lifetime cost of first-ever stroke care are approximately \$A100 000 per patient in Australia. $^{3-6}$ In a recent Northern Territory (NT) study, the estimated net lifetime healthcare cost for Aboriginal patients were $44 \%$ greater than for non-Aboriginal patients. $^{7}$

Over the past 25 years, improvement in stroke prevention and treatment has resulted in substantial increases in stroke survival. ${ }^{89}$ Although Aboriginal populations experience stroke at younger ages and have a higher prevalence of comorbidities, this improvement in survival was evident for Aboriginal 
and non-Aboriginal patients in the NT, although with much shorter survival in the Aboriginal patients after adjustments for age at onset and other confounders. ${ }^{10}$ Nationally, it has been recognised in the clinical guidelines that Aboriginal patients require access to general and stroke-specific care in rural and remote areas, consistent with their non-Aboriginal counterparts. ${ }^{11}$ However, in previous studies patients with stroke who were Aboriginal had less access to the hospital procedures or medications to prevent stroke than non-Aboriginal patients. ${ }^{12} 13$ Further, it has been identified in a national audit that Aboriginal patients with stroke received less access to recommended care, for example, none received intravenous thrombolysis or timely allied health assessments, they were less likely to be treated in a stroke unit, and subsequently experienced worse outcomes than non-Aboriginal patients. ${ }^{14}$ Further research is needed to understand the implications of these findings and verify, if in fact, disparities across the whole continuum of care exist, to inform policy and planning.

Cost-effectiveness of stroke care may be monitored using healthcare utilisation data to identify potential treatment biases. ${ }^{15}$ One important measure of cost-effectiveness is the marginal changes in health costs over the marginal changes in stroke survival. ${ }^{16}$ Cost-effectiveness analyses provide useful information to guide effective, efficient and equitable use of limited resources. ${ }^{15}$ Information on the cost-effectiveness of stroke care and prevention has been published previously. ${ }^{15}$ However, little is known about the cost-effectiveness of stroke care in Aboriginal compared with non-Aboriginal patients. ${ }^{18}$ The intuitive way in which cost-effectiveness analysis can handle both costs and effects simultaneously makes it a powerful tool for the evaluation of health policy and practice at a population level. ${ }^{19}$ The comparative cost-effectiveness design provides a valuable means to describe healthcare activities in a real-world settings relative to the outcome achieved. ${ }^{20}$

The NT is a large, sparsely populated area of northern Australia where a substantial Aboriginal population resides as opposed to other parts of Australia. In 2011, the NT resident population was 211947 (1\% of the Australian population), $27 \%$ of whom were Aboriginal Australians (nationally $2.5 \%) .{ }^{21}$ There are five public hospitals, which provided stroke care in the NT, and of which only one (Royal Darwin Hospital) has a specialised stroke unit, which was opened around 2008. Primary care for non-Aboriginal patients was generally provided by general practitioners (GPs) located in urban areas (herein referred to as urban-based primary care), whereas most of Aboriginal patients were from remote communities where primary care services were provided in remote clinics by nurses and Aboriginal health practitioners. In 2006, life expectancy at birth was 21 and 15 years shorter in Aboriginal than non-Aboriginal population for males ( 60 vs 81 years) and females (70 vs 85 years) respectively in the NT. ${ }^{22}$ Between 1999 and 2003, the burden of disease resulting from premature death and disability in the Aboriginal population was 2.8-3.3 times greater than in the non-Aboriginal population. ${ }^{23}$ The Aboriginal NT residents are more likely than their non-Aboriginal counterparts to suffer haemorrhagic stroke (HS) and comorbidities and die from a stroke. ${ }^{24}$ Despite there being significant improvements in stroke survival between 1992 and 2013, the hospital mortality was still $56 \%$ higher in Aboriginal than non-Aboriginal patients, after age adjustment. ${ }^{10}$ However, there was a lack of comprehensive assessments comparing Aboriginal and non-Aboriginal patients with stroke in terms of cost-effectiveness of stroke care including stroke specific procedures as recommended in clinical guidelines. ${ }^{11}$

The aim of the study was to compare the cost-effectiveness of stroke care for two populations with very different burdens of disease, the Aboriginal and non-Aboriginal Australians to explore whether inequalities in providing healthcare exist and to determine the efficiency of the healthcare that is provided. The study was designed to draw conclusions and make recommendations regarding cost-effectiveness of stroke care for Aboriginal patients.

\section{METHODS}

In undertaking this study, we adhered to the international guidelines for cost-effectiveness analysis. ${ }^{25}$ This is an observational cohort study based on data from 1 July 1992 to 30 June 2013 for Aboriginal and non-Aboriginal patients with first-ever stroke. Four administrative data sources were used. Individual patient-level data from the hospital inpatient data (HID) between 1982 and 2013 and event data from the primary care information system (PCIS) between 2009 and 2013, were merged using an encrypted unique patient identifier for patient tracking and survival analysis. Two additional data sources, Medicare and Pharmaceutical Benefits Scheme (PBS) data from 1993 to 2013, were used for calculating non-hospital costs.

Patients with stroke were identified from HID using the International Classifications of Diseases (ICD) version 9 to June 1998 and version 10 thereafter. Stroke was categorised into three types: HS (diagnosis codes 430, 431, 432.9 (ICD-9); I60, I61, I62.9 (ICD-10)), ischaemic stroke (IS) (433, 434; I63) and stroke type undetermined (UND) (436; I64). ${ }^{26}$ Transient ischaemic attack (TIA) (435.9; G45.9) was excluded from this study. Incidence of stroke was identified using the first-ever admission with a stroke diagnosis during the study period, and a stroke-free admission in the preceding clearance of at least 10 years using data from 1 July 1982. Follow-up time was calculated as the number of days between the first-ever admission date and the discharge date of the subsequent or recurrent admission. Each intervening hospital admission or readmission was regarded as a follow-up. Procedures including imaging, surgical, pharmaceutical, rehabilitation and other non-stroke specific procedures were defined by using the Australian Classification of Health Intervention (ACHI) block numbers. ${ }^{27}$ The numbers of 
ACHI coded procedures per hospitalisation were averaged for each patient. Follow-up time was censored at: the date of death for patients who died from causes other than stroke, or the discharge date of the last admission (or first admission if only admitted once) for patients discharged alive. Truncation was not used for censoring at the beginning nor the end of this study, ${ }^{28}$ because truncation might overestimate survival when resources were not available as part of rigorous follow-up and searches using the National Death Index (i.e. Australian national death registry).

Six main categories of service use were costed from a healthcare perspective: hospital inpatient care, outpatient, nursing home, primary care (GP and remote clinic), pharmaceuticals and allied health. Indirect (ie, loss of productivity), intangible (ie, loss on quality of life) and external social costs were beyond the scope of this study. Inpatient costs were calculated by multiplying the Australian national/refined diagnosis related group weights times the NT benchmark prices ${ }^{29}$ covering medical, nursing, supplies, imaging, pathology, allied health, pharmacy, critical care, operating room, emergency, prostheses, procedures, and hospital overhead oncosts. The stroke-related events (occasions of service) in remote communities were identified using PCIS data. The remote clinic costs were obtained by multiplying the number of events by the average cost estimate in the year the service was provided (eg, \$A36 in 2003). ${ }^{30}$ Since we did not have access to unit record GP data, the urban primary care cost was estimated as $3.2 \%$ of total NT Medicare benefits for GPs, because $3.2 \%$ of GP patients had a stroke or TIA according to the most recent GP survey. ${ }^{31}$ The PBS cost was estimated by the item codes for antihypertensive, antiplatelet and anticoagulant agents. The cost was multiplied by two to cover Section 100 (access to highly specialised drugs by remote Aboriginal people) expenditure and to counterbalance the under-coverage of data for drugs administered in remote areas, because preliminary analysis indicated that Section 100 expenditure was similar to the claimed PBS in the NT Aboriginal setting. Hospital costing methodology complies with the national guidelines for cost data collection ${ }^{29}$ and the cost estimate breakdowns have been reported elsewhere. ${ }^{7}$ Five per cent was applied to represent the present value of costs based on the reference year 2012/2013, because 5\% reflected an average level of health inflation. ${ }^{32}$

Case fatality was defined by using HID, where separation mode was recorded as deceased and stroke was recorded as a diagnosis at that episode. Health outcome was assessed through measuring survival time after stroke. Univariable analysis was performed using mean, median and the interquartile range (IQR) to describe demographics, time of incidence, hospitalisations, bed-days, numbers of procedures, costs and survival time. Chi-square significance test and Kaplan-Meier survival curves were used to compare Aboriginal and non-Aboriginal patients. Linear censored regression was used to adjust for loss to follow-up. Estimation of the incremental cost-effectiveness ratio (ICER) was adapted for comparing cost and survival for the Aboriginal and non-Aboriginal populations, based on the notion of maximising health gains with available resources. ${ }^{33}$ The bootstrap method with 2000 replications was used to construct the cost-effectiveness plane for assessment of the ICER variability. ${ }^{33} \mathrm{~A}$ threshold statistical value of $\$ \mathrm{~A} 120$ 000 per life-year was used to evaluate cost-effectiveness, as recommended by the Australian Safety and Compensation Council. ${ }^{34}$ The cost-effectiveness of healthcare for incident stroke was further analysed by using a marginal structural model (MSM) to account for time-dependent confounding and censoring. ${ }^{35}$ A $\log 10$-transformation was applied to the costs, because preliminary analysis showed the observed costs resembled a log-normal distribution. The multiplicative cost increment was used as an independent variable and survival as the dependent variable, because survival represents benefits of stroke care as an outcome, and the cost representing the resource use as the input. The hazard ratio (HR) of mortality for a unit of cost increment represents the proportional change in mortality hazard given the percent change in healthcare costs. A full range of thresholds for the ICER ( $\$ A 0-\$ A 500$ 000 per life-year) representing different values of willingness-to-pay was tested for assessing the uncertainty of the ICER. The willingness-to-pay acceptability curves were compared for comparative cost-effectiveness, that is, saving more life-years cost-effectively. Sensitivity analysis was also performed using 3\% and 10\% discount rates, with and without log-transformation.

The study was approved by the Human Research Ethics Committee of the NT Department of Health and the Menzies School of Health Research (HREC-2011-1680).

\section{RESULTS}

Between 1992 and 2013, 2889 patients were hospitalised with stroke in the NT, of whom 731 patients with recurrent stroke were excluded, leaving 2158 incident stroke cases in this study. Among incident cases, just over half were male $(54.3 \%)$, aged $<65$ years $(54.6 \%)$ or from non-remote areas $(55.2 \%)$, with $42.6 \%$ being IS, $29.8 \%$ HS and $46.0 \%$ of Aboriginal origin (table 1). The total median follow-up time was 318 days with IQR 13-1512 days. Aboriginal patients had 14 years younger age of stroke onset, $71.4 \%$ more hospital bed-days and $49.6 \%$ greater observed costs than non-Aboriginal patients, driven by a higher level of hospitalisations (median 4 vs 3) (all $\mathrm{p}<0.001)$. The average number of procedures per hospitalisation in Aboriginal patients was 6.9\% fewer than that for non-Aboriginal patients (2.7 vs 2.9, $\mathrm{p}<0.05)$. In particular, Aboriginal patients had significantly less imaging and rehabilitation procedures, but more other non-stroke specific procedures than the non-Aboriginal patients (all $\mathrm{p}<0.01$ ). Average numbers of surgical and pharmaceutical procedures were slightly fewer in the Aboriginal than non-Aboriginal patients with non-statistical significance $(0.36$ vs 0.41 and 0.14 vs 0.16 , respectively; both $\mathrm{p}>0.05)$. Number of incident 
Table 1 Characteristics of patients with stroke (n (\%)) by Aboriginality, Northern Territory, Australia, 1993-2013

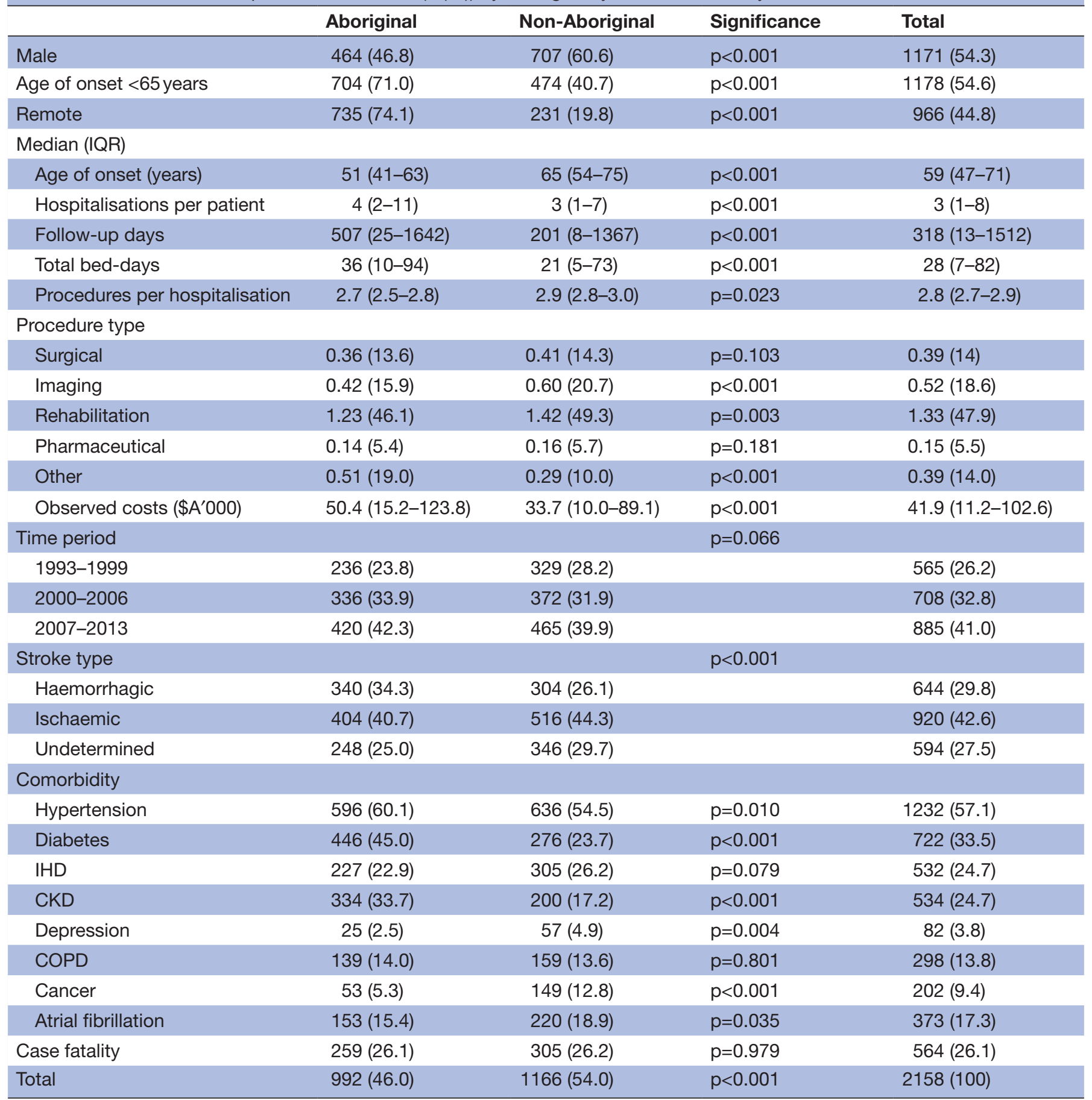

CKD, chronic kidney disease; COPD, chronic obstructive pulmonary disease; IHD, ischaemic heart disease.

strokes increased over time likely driven by population growth and ageing, despite there being no changes in the proportion between Aboriginal and non-Aboriginal patients. Compared with non-Aboriginal patients, Aboriginal patients were disproportionately more likely to experience HS, attributed to a greater prevalence of hypertension, diabetes and chronic kidney disease $(\mathrm{CKD})(\mathrm{p} \leq 0.01)$. There was no difference in total case fatality between Aboriginal and non-Aboriginal patients with stroke over the study period.

Figure 1 shows that there appeared to be slightly better survival in Aboriginal patients with stroke despite the log-rank test indicating statistical insignificance. Aboriginal patients were significantly younger. Age stratified analysis in figure 1B-D shows a slightly greater Aboriginal survival in the aged $65+$ group $(\mathrm{p}<0.05)$, but there were 
(A) All strokes

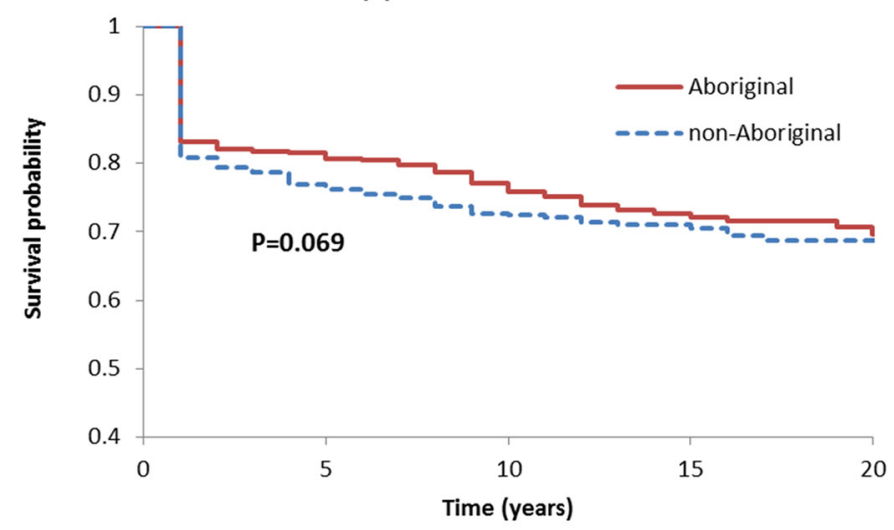

(C) Age between 45 and 64 years

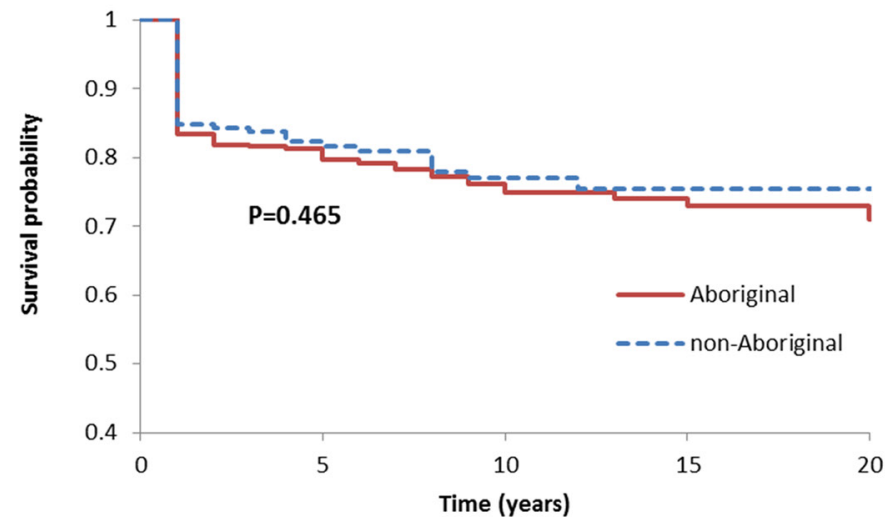

Figure 1 Survival by age and Aboriginality.

no significant Aboriginal survival differences in the other age groups.

Details of the average survival time and costs by stroke type comparing Aboriginal and non-Aboriginal patients are provided in table 2. Aboriginal patients tended to have better survival and greater costs than non-Aboriginal patients, especially after an IS $(\mathrm{p}<0.05)$. Overall, stroke costs were $20.5 \%$ more in Aboriginal than non-Aboriginal patients $(\mathrm{p}<0.001)$. The ICER of stroke care for Aboriginal patients compared with that for non-Aboriginal
(B) Age $<45$ years

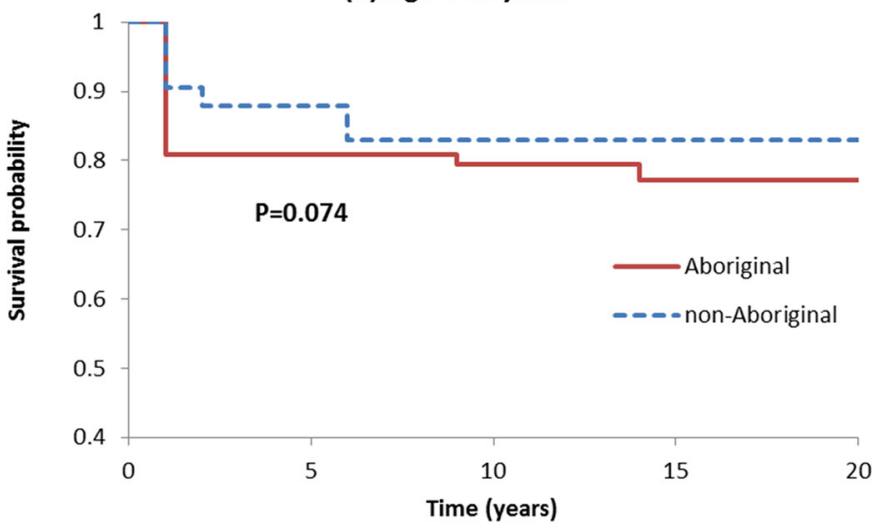

(D) Age $>=65$ years

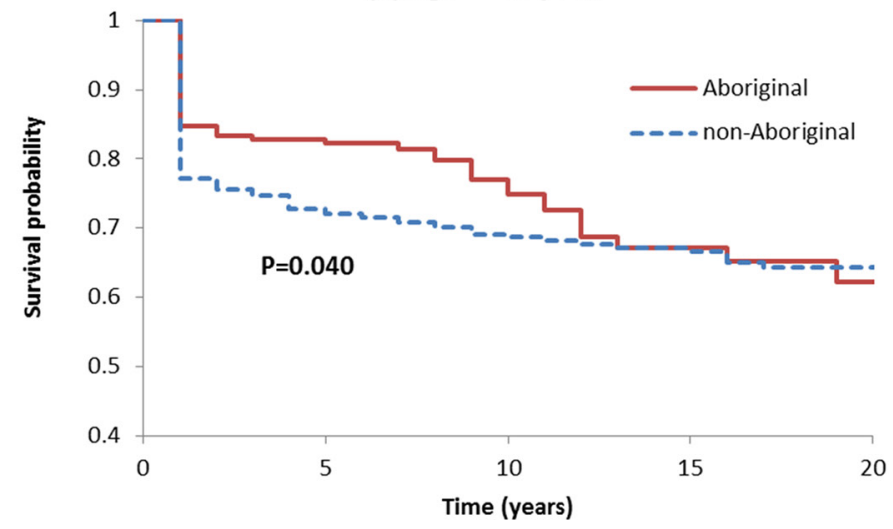

patients was an average of \$A110 965 per survival year, ranging from \$A69 163 in UND to \$A130 376 in HS (see bottom line in table 2 ). Figure 2 shows a great degree of uncertainty in both survival and costs, especially in UND, followed by IS and then HS, when comparing cost-effectiveness between Aboriginal and non-Aboriginal patients. It is unlikely that the greater uncertainty in UND was caused by the smaller sample size, because the bootstrap adjusts for sample size by resampling. ${ }^{33}$ The differential costs and effects were divided evenly across the threshold

Table 2 Average survival time and cost by stroke type and incremental cost-effectiveness ratio comparing Aboriginal and non-Aboriginal patients

\begin{tabular}{|c|c|c|c|c|}
\hline & HS & IS & UND & Total \\
\hline$n$ & 644 & 920 & 594 & 2158 \\
\hline \multicolumn{5}{|l|}{ Survival (years) } \\
\hline Non-Aboriginal & 5.9 & 9.9 & 9.7 & 8.6 \\
\hline p Value & 0.662 & 0.023 & 0.081 & 0.148 \\
\hline Aboriginal & 192675 & 448451 & 404800 & 331340 \\
\hline Non-Aboriginal & 166743 & 337732 & 310011 & 275045 \\
\hline p Value & 0.091 & $<0.001$ & 0.002 & $<0.001$ \\
\hline Net cost per life-year gained (ICER) & 130376 & 74343 & 69163 & 110965 \\
\hline
\end{tabular}

HS, haemorrhagic stroke; ICER, incremental cost-effectiveness ratio; IS, ischaemic stroke; UND, stroke type undetermined. 


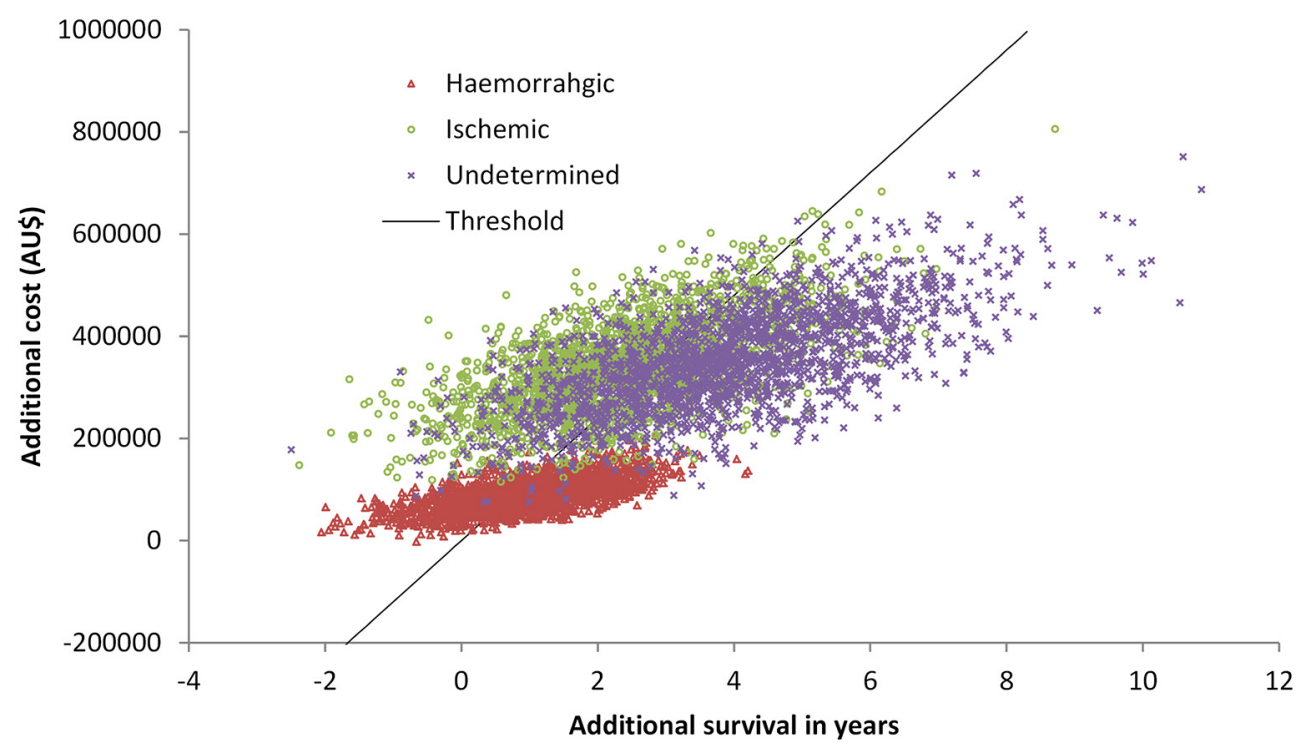

Figure 2 Cost-effectiveness plane comparing Aboriginal with non-Aboriginal patient by stroke type.

line, indicating that stroke care in Aboriginal patients was as cost-effective as in non-Aboriginal patients. Figure 3 provides the probability of whether the stroke care in Aboriginal patients would result in longer survival than in non-Aboriginal patients. The acceptability of achieving optimised stroke care in Aboriginal patients increased progressively with willingness-to-pay. At the threshold price (dotted line in figure 3), the probability of achieving an optimal cost-effectiveness in Aboriginal patients with UND was 0.76 , followed by HS (0.69) and IS (0.42).

After further adjustments for time-dependent confounders (time, age and comorbidities), time-independent confounders (sex and remoteness) and dependent censoring by using MSM (table 3), Aboriginal patients with stroke were $34.4 \%$ more likely to die of stroke than non-Aboriginal patients $(\mathrm{p}=0.008)$. Overall, HS was more than twice as likely to cause death as IS $(p<0.001)$, whereas there was no difference in survival between IS and UND. Stroke mortality increased with age at onset $(2.4 \%$ for every additional year of age, $\mathrm{p}<0.001)$,

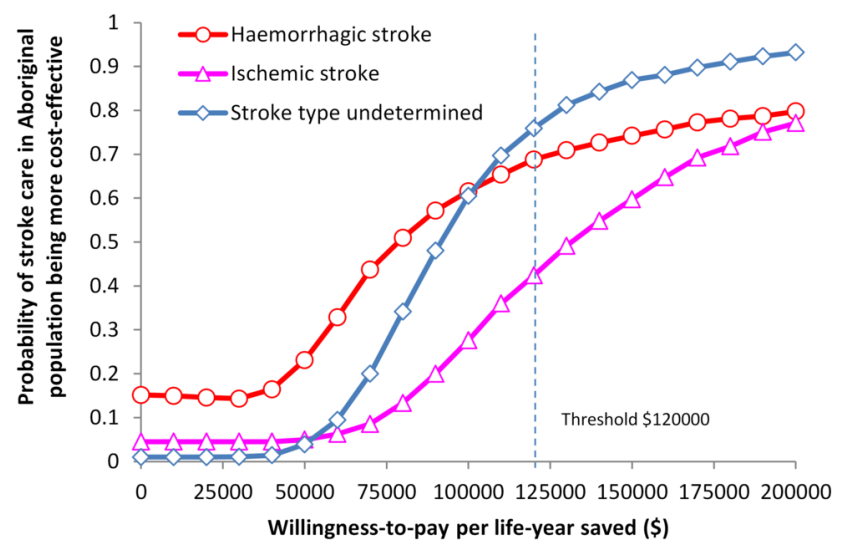

Figure 3 Acceptability curve for comparing costeffectiveness of stroke care between Aboriginal and nonAboriginal population. compounded by CKD $(\mathrm{p}<0.001)$ or cancers $(\mathrm{p}=0.011)$. The stroke mortality was reduced over time $(3.2 \%$ reduction annually, $\mathrm{p}<0.001$ ), and negatively correlated with residing in remote areas or having hypertension (both $\mathrm{p}<0.01$ ). In particular, stroke mortality was negatively associated with greater healthcare costs. A 10-fold increase in health costs was associated with a reduction in stroke mortality by $40.5 \%$ (HR=0.595, $\mathrm{p}<0.001)$. The stratified MSM by Aboriginality found that the HR was smaller among Aboriginal patients than in non-Aboriginal patients ( 0.525 vs 0.642$)$, indicating a slightly greater mortality reduction in Aboriginal, though statistically insignificant $(\mathrm{p}=0.25)$.

Sensitivity analysis provided evidence that the ICER was not dominant in favour of the Aboriginal population until the willingness-to-pay threshold was increased close to $\$$ A200 000 per life-year gained ( $>0.7$; figure 3 ). Probabilistic analyses confirmed that treating HS and UND were likely to be more cost-effective in Aboriginal patients than treating IS, likely related to underdiagnosis of stroke in remote areas. The average cost relativities were insensitive to differing discount rates. Log-transformation would generate more robust cost estimates. Without log-transformation, the magnitude of average costs might be affected, but the sign of difference between Aboriginal and non-Aboriginal patients remained consistent.

\section{Discussion}

In terms of survival outcome, to our knowledge, this is the first comparative cost-effectiveness evaluation providing evidence that stroke care among Aboriginal patients is as efficient as among the non-Aboriginal patients within the Australian context. Reports on the economic analysis of cost-effectiveness among Aboriginal and non-Aboriginal populations are rare. ${ }^{3637}$ In our sample, almost half $(46 \%)$ were Aboriginal patients. Overall, this percentage was disproportionate to the population proportion for the 
Table 3 Marginal structural proportional hazard model HR and $\mathrm{Cl}$ by Aboriginality

\begin{tabular}{|c|c|c|c|c|c|c|}
\hline & \multicolumn{2}{|c|}{ Aboriginal } & \multicolumn{2}{|c|}{ Non-Aboriginal } & \multicolumn{2}{|c|}{ All stroke } \\
\hline & HR & $95 \% \mathrm{Cl}$ & HR & $95 \% \mathrm{Cl}$ & HR & $95 \% \mathrm{Cl}$ \\
\hline Cost increment & 0.525 & 0.405 to 0.681 & 0.642 & 0.514 to 0.801 & 0.595 & 0.502 to 0.706 \\
\hline Ischaemic & 1.000 & - & 1.000 & - & 1.000 & - \\
\hline Haemorrhagic & 2.644 & 1.897 to 3.684 & 1.778 & 1.377 to 2.297 & 2.059 & 1.687 to 2.512 \\
\hline Time (year-1993) & 0.982 & 0.960 to 1.004 & 0.961 & 0.945 to 0.977 & 0.968 & 0.955 to 0.981 \\
\hline Age at onset (years) & 1.015 & 1.006 to 1.024 & 1.033 & 1.024 to 1.043 & 1.024 & 1.018 to 1.030 \\
\hline Female & 0.822 & 0.631 to 1.071 & 1.059 & 0.845 to 1.322 & 0.984 & 0.834 to 1.161 \\
\hline Remoteness & 0.826 & 0.629 to 1.086 & 0.727 & 0.527 to 1.002 & 0.753 & 0.615 to 0.921 \\
\hline Hypertension & 0.698 & 0.526 to 0.927 & 0.726 & 0.582 to 0.905 & 0.702 & 0.591 to 0.834 \\
\hline COPD & 1.017 & 0.696 to 1.735 & 0.989 & 0.725 to 1.348 & 1.014 & 0.773 to 1.330 \\
\hline Cancer & 1.299 & 0.524 to 3.217 & 1.534 & 1.090 to 2.158 & 1.503 & 1.098 to 2.058 \\
\hline Depression & - & - & 0.450 & 0.154 to 1.318 & 0.399 & 0.130 to 1.224 \\
\hline Atrial fibrillation & 1.200 & 0.751 to 1.917 & 0.989 & 0.705 to 1.388 & 1.099 & 0.841 to 1.437 \\
\hline
\end{tabular}

Cost increment represents per cent change in log(Cost).

CKD, chronic kidney disease; COPD, chronic obstructive pulmonary disease; IHD, ischaemic heart disease.

NT (27\% in 2011), reflecting the large impact stroke has on the Aboriginal population. Stroke care in the Aboriginal population may be even more beneficial because of the 14 years younger age at onset of stroke, resulting in more life-years potentially saved from the additional costs of providing care. Given that the majority of NT Aboriginal people live in rural or remote locations, it is important that ongoing efforts to support cardiovascular disease prevention and improve the access to best practice stroke services are prioritised.

There have been several estimates of the long-term cost of stroke in Australia in the past two decades, up to $\$ A 100$ $000,{ }^{3-6} 38$ mainly based on the North East Melbourne Stroke Incidence Study. ${ }^{3}$ These cost estimates were based on cohort studies conducted in urban areas and were likely to understate the NT patient lifetime costs, because of attrition bias caused by loss to follow-up and inability to account for the costs related to remote areas. ${ }^{39}$ In 2012/2013 Australian dollars, the estimated lifetime cost for an incident stroke in NT was \$A302 538 per patient between 1992 and 2013 after adjusting for loss to follow-up. ${ }^{7}$ Stroke cost was found to be $44 \%$ greater for Aboriginal patients. ${ }^{7}$

Accurate cost estimates are required for cost-effectiveness assessment related to improvements in stroke survival. ${ }^{40}$ Cost-effectiveness can be evaluated by comparing costs against outcomes between the alternative treatments. ${ }^{33}$ Understanding the cost drivers in stroke treatments informs health service decisions for cost-effective care. Most cost-effectiveness studies for stroke care were focused on specific procedures, ${ }^{17}{ }^{42}$ for example, pharmaceuticals, ${ }^{434}$ surgery, ${ }^{45} 46$ prevention ${ }^{47} 48$ and rehabilitation ${ }^{49}$; or evaluating alternative models of care ${ }^{50}$ for example, telemedicine ${ }^{51}$ and specialised stroke unit. $^{52} 53$ Hunter and colleagues evaluated the introduction of centralised stroke care, finding the service reduced mortality by $12 \%$ and saved more than $£ 811$ per patient-year. ${ }^{54}$ In Canada, comprehensive stroke care can reduce hospital admissions (3\%), bed-days $(26 \%)$, death $(15 \%)$ and nursing home care $(13 \%)$, as well as save costs of over CAD11 000 per stroke. ${ }^{55}$

Comparative cost-effectiveness is an emerging approach to comparing the costs and monitoring health outcomes of interventions and strategies to prevent, diagnose and treat diseases in real-world practice for informing clinical and policy decisions. ${ }^{56}{ }^{57}$ Previous reports suggested an effect of institutional bias in clinical decisions, which favoured stroke care in non-Aboriginal patients. ${ }^{12} 13$ Despite these reports, the cost-effectiveness of stroke care among different patient populations has not been investigated. Our study found that the uptake of hospital procedures by Aboriginal stroke patients with an incident stroke was $7 \%$ less than the non-Aboriginal patients (mainly imaging and rehabilitation). Univariable analysis showed that stroke care in Aboriginal patients is as cost-effective as in non-Aboriginal patients. The differential costs and effects were divided quite evenly across the willingness-to-pay threshold line. After taking into account the effects of patient-level confounders, time and loss to follow-up using MSM, we found that an increase in stroke 
care costs was associated with a slightly better health outcome for Aboriginal than non-Aboriginal patients with stroke, despite failing to reach statistical significance. Baker and colleagues evaluated cost-effectiveness of blood pressure control in kidney and cardiovascular disease treatment in an Aboriginal community. ${ }^{36}$ It was found that 3-year perindopril treatment was effective in delaying 1.5 years of haemodialysis per patient with a net annual cost of $\$ A 1200$, in comparison with a modelled historical control of the same Aboriginal population. Stroke was not identified in their study and non-Aboriginal controls were not compared. ${ }^{36}$ Grieve and colleagues compared stroke care costs and survival among different European countries and different ways of providing stroke care. ${ }^{19}$ The authors found that the cost-effectiveness may be related to specialised stroke care, which is required to provide interdisciplinary care after stroke. Our analysis directly compared cost-effectiveness of stroke care for both Aboriginal and non-Aboriginal patients. This study is also complemented by the use of MSM, which is more appropriate than conventional methods for managing censoring issues when estimating survival and costs. ${ }^{35}$

Strengths of this study include the large sample size of Aboriginal patients relative to the non-Aboriginal patients, access to data to ensure capture of incident cases and the ability to confidently link patient records across multiple sources of data. Further, the methodology in this study informs cost-effectiveness analysis for both patients and providers in real-world settings, which used non-randomised observational data and focused on more relevant health policy issues. ${ }^{56}{ }^{57}$ It is cost-effective to improve access for Aboriginal patients with suspected and confirmed stroke, especially with respect to imaging for rapid diagnosis and interdisciplinary rehabilitation to reduce the impacts of stroke. Upskilling of doctors and nurses in best practice stroke prevention and management is urgently required as inequities in access to optimal stroke care in different regions impact on the quality of care. ${ }^{258}$ It is important to develop specialist care in regional centres to improve the overall availability of best practice stroke care in regional and remote areas in Australia.

This study covered 21 years from 1992 to 2013, largely because of the requirements to identify sufficient incident cases and follow-up for survival analysis. Several limitations should be noted. First, the results in this study are most relevant to Aboriginal population living in remote locations, who experience socioeconomic disadvantage and high burden of disease in a high-income country. ${ }^{596}$ The stroke care cost-effectiveness results may be relevant to other disadvantaged populations. Second, our cost estimates may not be precise. PCIS was only available for Aboriginal communities after 2009. PCIS data were only used for costing primary care, which was extrapolated to cover the previous years and adjusted for health inflation. In this study, we used administrative HID to identify stroke cases and did not include minor and low-cost cases managed solely by outpatient department, GP or remote clinics. These low-cost cases would comprise a small proportion of stokes (previously estimated at $12 \%$ in 1997). ${ }^{3}$ A top-down approach was applied to calculate the GP, nursing home and allied health costs, which might lead to overestimation or underestimation of the true costs. Various statistical methods were used to assess the robustness of the point estimates. More research is needed to further explore the cost-effectiveness of stroke care using prospective patient-level costing data. Third, another potential source of uncertainty in cost-effectiveness evaluation was the lack of non-NT or non-stroke controls, assuming stroke care as a whole is cost-effective in the NT, and stroke survival was independently associated with costs of stroke care and perceived confounders. Fourth, we were unable to measure levels of disability and quality of life among stroke survivors. It might be that there were important differences in levels of stroke-related disabilities between Aboriginal and non-Aboriginal patients. Finally, the joint effects of multiple comorbidities and their interactions were not considered in this study due to limited sample sizes.

In conclusion, stroke care for Aboriginal patients is at least as cost-effective as for non-Aboriginal patients managed within a sparsely populated but geographically large region of Australia, where healthcare resources are limited. Stroke care was found to present worthwhile and equitable survival benefits for Aboriginal patients in remote communities, notwithstanding their higher burden of disease. These data may provide useful information for other countries with Indigenous populations living in regions with similar geographical and resource constraints.

Author affiliations

${ }^{1}$ Health Gains Planning, Northern Territory Department of Health, Darwin, Northern Territory, Australia

${ }^{2}$ Menzies School of Health Research, Darwin, Northern Territory, Australia

${ }^{3}$ Royal Darwin Hospital, Darwin, Northern Territory, Australia

${ }^{4}$ Department of Endocrinology, Metabolism and Diabetes, Karolinska University Hospital, Stockholm, Sweden

${ }^{5}$ Department of Molecular Medicine and Surgery, Karolinska Institutet, Stockholm, Sweden

${ }^{6}$ Stroke and Ageing Research, School of Clinical Sciences, Monash University, Clayton, Victoria, Australia

${ }^{7}$ Stroke Division, The Florey Institute of Neuroscience and Mental Health, University of Melbourne, Melbourne, Victoria, Australia

${ }^{8}$ Centre for Population Health Research, Deakin University, Geelong, Victoria, Australia

Acknowledgements The authors thank the Northern Territory Department of Health for sponsoring this project.

Contributors YZ designed the study, collected costing data, undertook data linkage and statistical analysis and wrote the first draft of the manuscript. SG, HFa, HFI and DAC participated in the literature review, methodology development, discussion and revision of the manuscript and contributed equally to this work.

Funding HFa was supported by the Magnus Bergvall Foundation, Karolinska Institutet and the Stockholm County Council. DAC held a National Health and Medical Research Council (NHMRC) Research Fellowship cofunded with Heart Foundation (1063761).

Competing interests None declared.

Ethics approval Human Research Ethics Committee of the NT Department of Health and the Menzies School of Health Research. 
Provenance and peer review Not commissioned; externally peer reviewed.

Data sharing statement No additional data are available.

Open Access This is an Open Access article distributed in accordance with the Creative Commons Attribution Non Commercial (CC BY-NC 4.0) license, which permits others to distribute, remix, adapt, build upon this work non-commercially, and license their derivative works on different terms, provided the original work is properly cited and the use is non-commercial. See: http://creativecommons.org/ licenses/by-nc/4.0/

(C) Article author(s) (or their employer(s) unless otherwise stated in the text of the article) 2017. All rights reserved. No commercial use is permitted unless otherwise expressly granted.

\section{REFERENCES}

1. Feigin VL, Forouzanfar $\mathrm{MH}$, Krishnamurthi R, et al. Global and regional burden of stroke during 1990-2010: findings from the global burden of disease study 2010. Lancet 2014;383:245-55.

2. National Stroke Foundation. Stroke in Australia: No Postcode Untouched. Australia: NSF, 2014

3. Dewey HM, Thrift AG, Mihalopoulos C, et al. Cost of stroke in Australia from a societal perspective: results from the North East Melbourne Stroke Incidence Study (NEMESIS). Stroke 2001;32:2409-16.

4. Cadilhac DA, Carter R, Thrift AG, et al. Estimating the long-term costs of ischemic and hemorrhagic stroke for Australia: new evidence derived from the North East Melbourne Stroke Incidence Study (NEMESIS). Stroke 2009;40:915-21.

5. Gloede TD, Halbach SM, Thrift AG, et al. Long-term costs of stroke using 10-year longitudinal data from the North East Melbourne Stroke Incidence Study. Stroke 2014;45:3389-94.

6. Deloitte Access Economics. The Economic Impact of Stroke in Australia. Australia: National Stroke Foundation, 2013.

7. Zhao Y, Condon J, Lawton P, et al. Lifetime direct costs of stroke for indigenous patients adjusted for comorbidities. Neurology 2016;87:458-65.

8. Carter KN, Anderson CS, Hackett ML, et al. Improved survival after stroke: is admission to hospital the major explanation? Trend analyses of the Auckland Regional Community Stroke Studies. Cerebrovasc Dis 2007;23:162-8.

9. Yang Q, Botto LD, Erickson JD, et al. Improvement in stroke mortality in Canada and the United States, 1990 to 2002. Circulation 2006;113:1335-43.

10. Zhao Y, Condon J, You J, et al. Assessing improvements in survival for stroke patients in the Northern Territory 1992-2013: a marginal structural analysis. Aust Health Rev 2015;39:437-43.

11. Stroke Foundation Clinical Guidelines for Stroke Management 2010. Melbourne: Stroke Foundation, 2010.

12. Pepper EM, Cadilhac DA, Pearce DC, et al. Stroke among indigenous Australians at Royal Darwin Hospital, 2001-02. Med J Aust 2006;184:195.

13. Cunningham J. Diagnostic and therapeutic procedures among Australian hospital patients identified as indigenous. Med J Aust 2002;176:58-62.

14. Kilkenny MF, Harris DM, Ritchie EA, et al. Hospital management and outcomes of stroke in indigenous Australians: evidence from the 2009 Acute Care National Stroke Audit. Int J Stroke 2013;8:164-71.

15. Cobiac LJ, Magnus A, Barendregt JJ, et al. Improving the costeffectiveness of cardiovascular disease prevention in Australia: a modelling study. BMC Public Health 2012;12:398.

16. Hoffman A, Pearson SD. 'Marginal medicine': targeting comparative effectiveness research to reduce waste. Health Aff 2009;28:w710-w718.

17. Holloway RG, Benesch CG, Rahilly CR, et al. A systematic review of cost-effectiveness research of stroke evaluation and treatment. Stroke 1999;30:1340-9.

18. Katzenellenbogen JM, Vos T, Somerford P, et al. Burden of stroke in indigenous Western Australians: a study using data linkage. Stroke 2011:42:1515-21.

19. Grieve R, Hutton J, Bhalla A, et al. A comparison of the costs and survival of hospital-admitted stroke patients across Europe. Stroke 2001;32:1684-91.

20. US Department of Health and Human Services. Federal Coordinating Council for Comparative Effectiveness Research: Report to the President and Congress. Washington, DC: US Department of Health and Human Services, 2009.

21. Australian Bureau of Statistics. 2011 Census Quickstats. Canberra: ABS, 2013. http://www.abs.gov.au/websitedbs/censushome.nsf/ home/quickstats?opendocument\&navpos=220 (accessed $29 \mathrm{Dec}$ 2015).

22. Tay EL, Li SQ, Guthridge S. Mortality in the Northern Territory, 19672006. Darwin: Department of Health, 2013.

23. Zhao Y, You J, Guthridge S. Burden of Disease and Injury in the Northern Territory, 1999-2003. Darwin: Department of Health and Families 2009.

24. You J, Condon JR, Zhao Y, et al. Stroke incidence and case-fatality among indigenous and non-indigenous populations in the Northern Territory of Australia, 1999-2011. Int J Stroke 2015;10:716-22.

25. World Health Organization. Making Choices in Health: WHO Guide to Cost-Effectiveness Analysis. Geneva: WHO, 2003.

26. Harris D, Cadilhac D, Hankey G, et al. National stroke audit: the Australian experience. Clinical Audit [E] 2010;2:25-31.

27. National Centre for Classification in Health. Australian Classification of Health Interventions (ACHI). Sydney: University of Sydney, 2008.

28. Gail MH, Benichou J. Encyclopedia of Epidemiologic Methods: Wiley, 2000.

29. Independent Hospital Pricing Authority. National Hospital Cost Data Collection (NHCDC). Sydney: IHPA, 2014. http://www.aihw.gov.au/ WorkArea/DownloadAsset.aspx?id=60129547086 (accessed 29 Dec 2015).

30. Zhao Y, Hanssens P, Byron P, et al; Cost Estimates of Primary Health Care Activities for Remote Aboriginal Communities in the Northern Territory. Darwin: Department of Health and Community Services, 2006.

31. Britt H, Miller GC, Henderson J, et al; General Practice Activity in Australia 2013-14: BEACH Bettering the Evaluation and Care of Health. Sydney: Sydney University Press, 2014.

32. Australian Bureau of Statistics. Consumer Price Index, Australia. Canberra: ABS, 2015. http://www.abs.gov.au/ausstats/abs@.nsf/mf/ 6401.0 (accessed 29 Dec 2015)

33. Drummond MF, Sculpher MJ, Torrance GW, et al. Methods for the Economic Evaluation of Health Care Programmes. Oxford: Oxford University Press, 2005.

34. Australian Safety and Compensation Council. The Health of Nations: the Value of a Statistical Life. Canberra: Commonwealth of Australia, 2008.

35. Faries DE, Kadziola ZA. Analysis of longitudinal observational data using marginal structural models. In: Faries DE, Leon AC, Haro JM, Obenchain R. eds. Analysis of Observational Health Care Data using SAS. North Carolina: SAS, 2014.

36. Baker PR, Hoy WE, Thomas RE. Cost-effectiveness analysis of a kidney and cardiovascular disease treatment program in an Australian aboriginal population. Adv Chronic Kidney Dis 2005;12:22-31.

37. Young TK. Review of research on aboriginal populations in Canada: relevance to their health needs. BMJ 2003;327:419-22.

38. Dewey HM, Thrift AG, Mihalopoulos C, et al. Lifetime cost of stroke subtypes in Australia: findings from the North East Melbourne Stroke Incidence Study (NEMESIS). Stroke 2003;34:2502-7.

39. Lin DY, Feuer EJ, Etzioni R, et al. Estimating medical costs from incomplete follow-up data. Biometrics 1997;53:419-34.

40. Owiti EA. Cost Effectiveness and Survival Analysis of HIV and AIDS Treatment in Kenya. Kenya: University of Nairobi, 2013.

41. Griffiths RI, Gleeson ML, Mikhael J, et al. Impact on medical cost, cumulative survival, and cost-effectiveness of adding rituximab to first-line chemotherapy for follicular lymphoma in elderly patients: an observational cohort study based on SEER-Medicare. J Cancer Epidemiol 2012;978391:1-13.

42. Demaerschalk BM, Hwang HM, Leung G. Cost analysis review of stroke centers, telestroke, and rt-PA. Am J Manag Care 2010;16:537-44.

43. Gage BF, Cardinalli AB, Albers GW, et al. Cost-effectiveness of warfarin and aspirin for prophylaxis of stroke in patients with nonvalvular atrial fibrillation. JAMA 1995;274:1839-45.

44. Lightowlers S, McGuire A. Cost-effectiveness of anticoagulation in nonrheumatic atrial fibrillation in the primary prevention of ischemic stroke. Stroke 1998;29:1827-32.

45. Cronenwett JL, Birkmeyer JD, Nackman GB, et al. Costeffectiveness of carotid endarterectomy in asymptomatic patients. $J$ Vasc Surg 1997;25:298-311.

46. Kuntz KM, Kent KC. Is carotid endarterectomy cost-effective? an analysis of symptomatic and asymptomatic patients. Circulation 1996;94:II194-8.

47. Cadilhac DA, Carter R, Thrift AG, et al. Organized blood pressure control programs to prevent stroke in Australia: would they be costeffective? Stroke 2012;43:1370-5.

48. Freeman JV, Zhu RP, Owens DK, et al. Cost-effectiveness of dabigatran compared with warfarin for stroke prevention in atrial fibrillation. Ann Intern Med 2011;154:1-11. 
49. Widén Holmqvist L, von Koch L, Kostulas V, et al. A randomized controlled trial of rehabilitation at home after stroke in southwest Stockholm. Stroke 1998;29:591-7.

50. Patel A, Knapp M, Perez I, et al. Alternative strategies for stroke care: cost-effectiveness and cost-utility analyses from a prospective randomized controlled trial. Stroke 2004;35:196-203.

51. LaMonte MP, Bahouth MN, Xiao Y, et al. Outcomes from a comprehensive stroke telemedicine program. Telemed J E Health 2008;14:339-44.

52. Launois R, Giroud M, Mégnigbêto AC, et al. Estimating the costeffectiveness of stroke units in France compared with conventional care. Stroke 2004;35:770-5.

53. Saka O, Serra V, Samyshkin Y, et al. Cost-effectiveness of stroke unit care followed by early supported discharge. Stroke 2009;40:24-9.

54. Hunter RM, Davie C, Rudd A, et al. Impact on clinical and cost outcomes of a centralized approach to acute stroke care in London: a comparative effectiveness before and after model. PLOS One 2013;8:e70420.
55. Krueger $\mathrm{H}$, Lindsay $\mathrm{P}$, Cote $\mathrm{R}$, et al. Cost avoidance associated with optimal stroke care in Canada. Stroke 2012;43:2198-206.

56. Berger ML, Dreyer N, Anderson F, et al. Prospective observational studies to assess comparative effectiveness: the ISPOR good research practices task force report. Value Health 2012;15:217-30.

57. Dreyer NA, Schneeweiss S, McNeil BJ, et al. GRACE principles: recognizing high-quality observational studies of comparative effectiveness. Am J Manag Care 2010;16:467-71.

58. National Stroke Foundation. National Stroke Audit: Rehabilitation Services Report. Australia: NSF. 2016.

59. Zhao Y, Malyon R. Cost drivers of remote clinics: remoteness and population size. Aust Health Rev 2010;34:101-5.

60. Zhao Y, Condon JR, Guthridge S, et al. Living longer with a greater health burden--changes in the burden of disease and injury in the Northern Territory indigenous population between 19941998 and 1999-2003. Aust N Z J Public Health 2010;34 Suppl 1:S93-S98. 